\title{
SYNTHESIS AND CHARACTERIZATION \\ OFGOLDNANOPARTICLESUSING CEFOTAXIMSODIUM AND ITS SCHIFF BASE DERIVATIVE WITH 4-N,N-DIMETHYLAMINO BENSALDEHYDEAS REDUCING ANDSTABILIZING AGENTS
}

\author{
AhlamJameelAbdulghani ${ }^{\star}$, RashaKudherHussain \\ Chemistry Department, College of Science, Baghdad University, Baghdad, Iraq \\ ${ }^{*}$ Corresponding author, \\ Email: ahlamjameel@scbaghdad.edu.iq
}

\begin{abstract}
Conjugated GNPs were synthesized by one step reaction of $\mathrm{AuCl}_{4}{ }^{-}$with cefotaxim (CFX) and its Schiff base derivative with 4-N,N-dimethylamino benzaldehyde [sodium 3-(acetoxymethyl)-7-((2Z)-2-(2-(4-dimethylamino) benzylideneamino) thiazol-4-yl)-2-(methoxyimino) acetamido)-8-oxo-5-thia-1-azabicyclo[4.2.0]oct-2-ene-2-carboxylate] (SCFX)without the need of reducing and functionalizing agents. The reduction process was monitored by uv-visible spectrophotometry and the synthesized GNPs were characterized by TEM, SEM, AFM and XRD analysis. The conjugation of GNPs with the two ligands was characterized by FTIR spectrophotometry. The size, morphology, and stability of GNPs were varied with concentration ratio of ligand/Au(III), $\mathrm{pH}$ medium and reaction temperature
\end{abstract}

Keywords:-Gold nanoparticles; cefotaxim; Schiff base; AFM; TEM,

\section{Council for Innovative Research}

Peer Review Research Publishing System

\section{Journal: Journal of Advances in Chemistry}

Vol. 11, No. 8

editorjaconline@gmail.com, www.cirjac.com 


\section{INTRODUCTION}

Several methods have been reported to conjugate anti-biotics with GNPs [1-6].Highly surface area of nanoparticles increases drug density on the surface and allows higher concentrations of drug or multiple drugs loaded on single particles $[7,8]$.The Synthesis of GNPs can be proceeded in a one-step reaction where the antibiotic act as a reducing and functionalizing agent to afford the antibiotic- functionalized nanomaterial without using any linkers [4-6]. Cepalosporins are $\beta$ - lactam antibiotics which are based upon the four-membered nitrogen-containing beta-lactam ring that gives these agents their antibacterial activity. Stable GNPs were synthesized by one step reaction using different cephalosporin antibiotics such as cephalexin , cefaclor, [3] ciproflaxin [1], and ceftriaxone[1] as a stabilizing agents in presence of reducing agent. As aprimary amine group carriers, some of these drugs acted as both reducing and capping agent for the synthesis of gold nanoparticles[1-3,7].Accordingly, condensation of the free amino group of $\beta$-lactam antibiotics with different carbonyl compounds to form Schiff bases may inhibit the reduction of Au(III) ions. In this paper, we describe a single one pot synthesis of gold nanoparticles using the $\beta$-lactam antibiotic, cefotaxim, (CFX) compared with its Schiff base derivative [sodium 3-(acetoxymethyl)-7-((2Z)-2-(2-(4-dimethylamino) benzylideneamino) thiazol-4-yl)2-(methoxyimino) acetamido)-8-oxo-5-thia-1-azabicyclo[4.2.0]oct-2-ene-2-carboxylate] (SCFX)in aqueous solutions without the addition of reducing ordispersing agents.

The reduction process was pursued by uv-visible spectrophotometry at different conditionslike concentration ratio of reactants, $\mathrm{pH}$ and temperature. The synthesized GNPs were characterized by uv-visible spectrophotometry, transmission electron microscopy (TEM), scanning electron microscopy (SEM), atomic force microscopy(AFM) and by X-ray diffraction (XRD) analysis

\section{EXPERIMENTAL}

\subsection{Instrumentation}

Electronic spectra in the (UV-Visible) region (200-1100) $\mathrm{nm}$ were recorded on SHIMADZU 1650 UV-Visible spectrophotometer. FTIR spectra were recorded on Shimadzu FT-IR 8400 S Fourier transform using KBr and CsI discs. TEM images were acquired using Philips CM10 Transmission electron microscope. Samples for TEM studies were prepared by placing a drop of the gold colloidal solution on a carbon coated copper grid which was driedunder vacuum. SEM images were acquired using TE SCAN VEGA III, Czech. Scanning electron microscope.AFM images were acquired using AFM model AA 3000 SPM 220 V-Angstrum Advanced INC. USA. Samples were prepared by applying few drops GNP solutions on a glass slide and a thin layer was formed as uniform as possible followed by vacuum drying. X-Ray diffraction (XRD) measurements were performed using a Shimadzu XRD-6000 x-ray diffraction spectrometer. All GNPs solutions were dialyzed prior to analysis by using a cellulose tube (MW cutoff $12400 \mathrm{D}$ ) against $1 \mathrm{~L}$ of DDW for $9 \mathrm{~h}$ at 30 ${ }^{\circ} \mathrm{C}$. Separation of nanoparticles was achieved by centrifugation CENTERFUGE C $417800 \quad 14000$ r.p.m., Jouan, (France).

\subsection{Materials and methods}

All the following chemicals were of analytical grades and were used as received from suppliers : Cefotaxime Sodium $\mathrm{C}_{16} \mathrm{H}_{16} \mathrm{~N}_{5} \mathrm{O}_{7} \mathrm{~S}_{2} \mathrm{Na} \quad \mathrm{GMBH}$,sodium chloroauratedihydrate $\mathrm{NaAuCl}_{4} .2 \mathrm{H}_{2} \mathrm{O}$ (Analar BDH),potassium hydrogen phthalate2-( $\left.\mathrm{HO}_{2} \mathrm{C}\right) \mathrm{C}_{6} \mathrm{H}_{4} \mathrm{CO}_{2} \mathrm{~K}, 99.95 \%(\mathrm{BDH})$, potassium chloride, potassium dihydrogen orthophosphate $\mathrm{KH}_{2} \mathrm{PO}_{4}$, and dipotassium hydrogen orthophosphate 99\% (Analar). The Schiff base ligandSCFXwas prepared before and the structural characterization of the compound wasreported in our previous work [8]. Standard solutions of cefotaxime sodium salt $\left(\mathrm{C}_{16} \mathrm{H}_{16} \mathrm{~N}_{5} \mathrm{O}_{7} \mathrm{~S}_{2} \mathrm{Na}, 2 \times 10^{-4} \mathrm{M}\right)$, and SCFX $\left(1.6 \times 10^{-3} \mathrm{M}\right)$ werepreparedbydissolving0.1000gof each reactant in

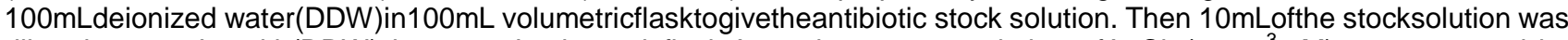
diluted to $100 \mathrm{~mL}$ with(DDW) ina $100 \mathrm{~mL}$ volumetricflask.A stock aqueous solution of $\mathrm{AuCl}_{4}^{-}\left(5 \times 10^{-3} \mathrm{M}\right)$ was prepared by dissolving $0.1000 \mathrm{~g}$ of $\mathrm{NaAuCl}_{4} \cdot 2 \mathrm{H}_{2} \mathrm{O}$ in $50 \mathrm{~mL}$ DDW in a volumetric flask. A standard solution of $\mathrm{AuCl}_{4}^{-1}\left(5 \times 10^{-4} \mathrm{M}\right)$ which contains $\left(2.5 \times 10^{-4} \mathrm{M}\right)$ of $\mathrm{Au}^{3+}$ ions, was prepared by diluting $10 \mathrm{~mL}$ of the stock solution to $100 \mathrm{ml}$ with (DDW).

\section{Synthesis of gold nanoparticle conjugates}

To different volumes of $\mathrm{AuCl}_{4}^{-}$standard solutions $\left(5 \times 10^{-4} \mathrm{M}\right):(0.25,0.5,0.75,1.0,1.25,1.5,2.0,2.5,3.0$, and 3.5 $\mathrm{mL}$ ) in ten $5 \mathrm{~mL}$ volumetric flasks were added one $\mathrm{mL}$ aliquots of standard solution ( 2 and $\left.1.6 \times 10^{-4} \mathrm{M}\right)$ of each of cefotaxime andSCFX respectively with continuous stirring. The volumes were then completed to $5 \mathrm{~mL}$ by DDW, and the absorbance of each solution was measured at room temperature at different time intervals. To study the effect of $\mathrm{pH}$ the synthesis of GNps was carried out at different pH $(2.4,2.89,3.84,4.85,5.8,6.92,7.55,8.92,9.9,10.9,11.9)$. To study 
the effect of temperature on the synthesis of GNPs by each ligand each set of solutions was heated for 5 minutes at ( 30 , $40,50,60,70$ and $80^{\circ} \mathrm{C}$ ) for $5 \mathrm{~min}$, in water bath with continuous stirring. The effect of heating periods on the synthesis rate of GNPs were studied by heating each sample at the selectedtemperature for $(5,10,15,20,25,30,45,60) \mathrm{min}$.

\section{RESULTS AND DISCUSSION}

The UV-visible spectra of cefotaxime (CFX), SCFX and $\mathrm{AuCl}_{4}^{-}$solutions as well as GNP solutions prepared from mixing equal volumes of standard aqueous solutions of $\mathrm{AuCl}_{4}^{-}$(III) with each ligand at room temperature are shown in (Figure 1). The spectrum of $\mathrm{AuCl}_{4}^{-}$solution exhibited a high intensity band at $\lambda 240 \mathrm{~nm}$ and with a shoulder $\lambda 290$ $\mathrm{nm}$ assigned to LMCT transitions of tetrachloroaurate complex [9,10]. The spectrum of the (CFX) antibiotic (Figure 1A)) displayed a multiplet covering the uv region at wavelength range 200-300 nm with three absorption maxima at $\lambda 238,259$ and $297 \mathrm{~nm}$ assigned to the $\Pi \rightarrow \pi^{*}$ transition [11]. The spectrum of SCFX in DDW (Figure 1B) displayed a multiplet band with three absorption peaks located at $\lambda_{\max } 235,295$, and $342 \mathrm{~nm}$ attributed to the $\pi \rightarrow \pi^{*}$ transition and a low intensity band observed at $\lambda_{\max } 430 \mathrm{~nm}$ refers to the $\mathrm{n} \rightarrow \pi^{*}$ transition[11]. The solution mixtures of $\mathrm{AuCl}_{4}^{-}$with the two ligands showed pink red colors and their spectra exhibited single absorption bands at $\lambda_{\max } 531$ and $532 \mathrm{~nm}$ respectively, indicating the reduction of $\mathrm{AuCl}_{4}{ }^{-}$by the two ligands to form GNPs. The time required for the development of the pink color was 2 and and $3.5 \mathrm{~h}$ for SCFX andCFX respectively
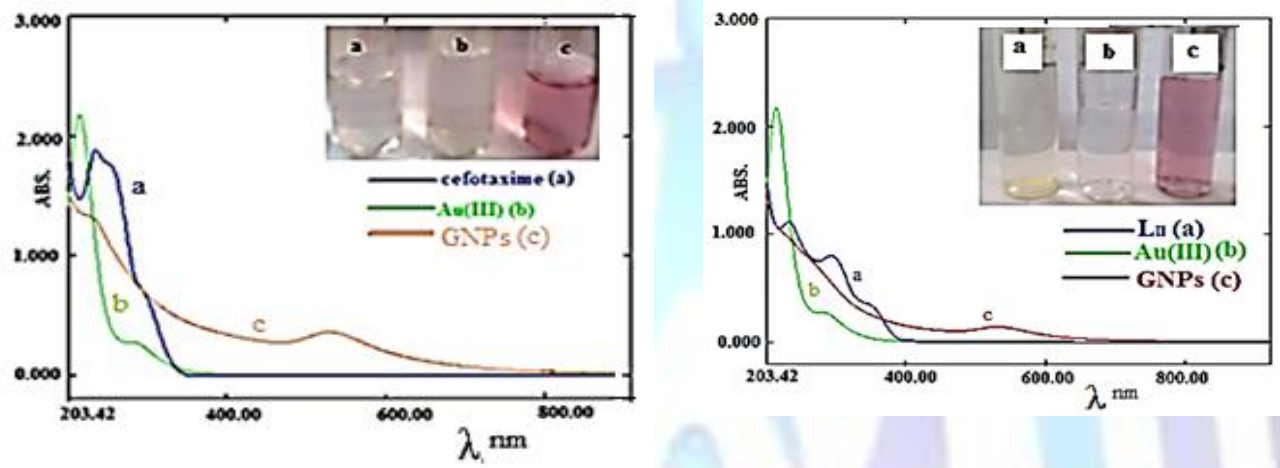

Figure 1. The UV-visible spectra and images of a- cefotaxime b- $\mathrm{AuCl}_{4}{ }^{-}$and c-synthesized gold nanoparticle in aqueous solutions after $24 \mathrm{~h}$.

No additional bands were observed in the visible or NIR region which refers to the formation of spherical gold nanoparticles[12-20]. The proposed mechanism suggests that formation of GNPs may have proceeded through the oxidation of the amine of the aromatic thiazole ring from which the formed electrons go on to reduce the auric(III) ions to gold atoms according to the literature[21,22]. Cefotaxim molecules contain many active groups such as amino, amido, thio and carboxyl groups, which react easily with GNPs by chelation [23]. Furthermore, Cefotaxime molecules themselves, like many beta lactam drugs, can bind each other through van der Waals interaction and hydrogen bonding [24]. Ultimately, assemblies are made up of nanogold cores and the surrounding cefotaxime molecules. The higher capability of the Schiff base ligands SCFX to reduce the Au(III) ions to GNPs compared with CFX indicatesthe presence of additional site for electron donation or for further stabilization of GNPs like the azomethinegroup.It also proves that the reduction process by (CFX) is not attributed to amino group of thiazole ring only, but other electron donor sites at the beta lactam ring, amide group moiety as well as thio and carboxyl groups, at the thiazine ring are involved in the reduction process and chelation with GNPs[25].

\subsection{Characterization of prepared gold nanoparticles}

The size, morphology and surfaces of the synthesized gold nanoparticles were investigated by SEM, TEM and AFM analysis of GNPs. The TEM analysis of the cefotaxime conjugated GNPs showed that the particles were spherical in shape with smooth surface morphology and mono-dispersed in nature with narrow size distribution and mean size diameters of around $17 \mathrm{~nm}$ (Figures2). The SEM images and size distribution for cefotaxime and SCFX conjugated GNPs (Figures3 and 4 respectively) showed spherical nanoparticleswith average size diameters around 60 and $27 \mathrm{~nm}$ respectively which indicates the difference in stabilizing effect between the two ligands. However the AFM images (Figures5 a and b respectively) showed the opposite result as the GNPs were spherical in shape and the average particle diameters were around 56 and $66 \mathrm{~nm}$, respectively, which may be attributed to different sample preparation conditions that caused the formation of agglomerates. 


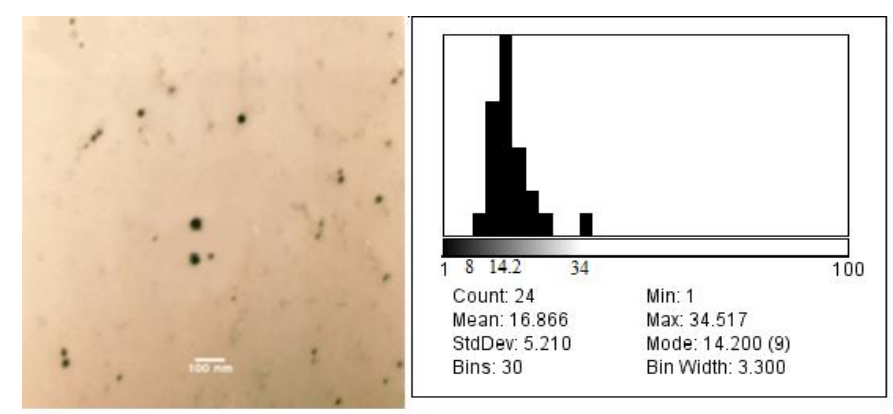

Figure2. TEM image and particle size distribution for CFX- GNPs conjugates.
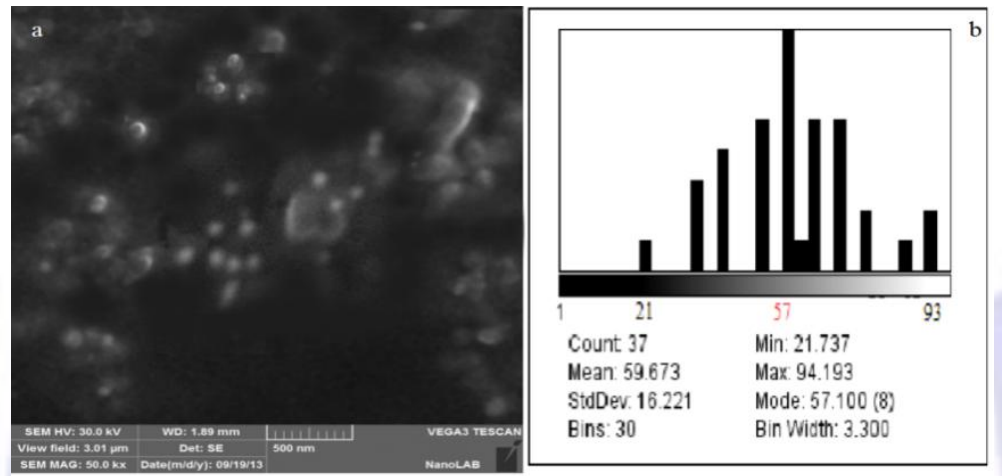

Figure 3.a-SEM image b-particle size distribution of the cefotaxime- synthesized GNPs (average particle size diameter $=60 \mathrm{~nm}$ )
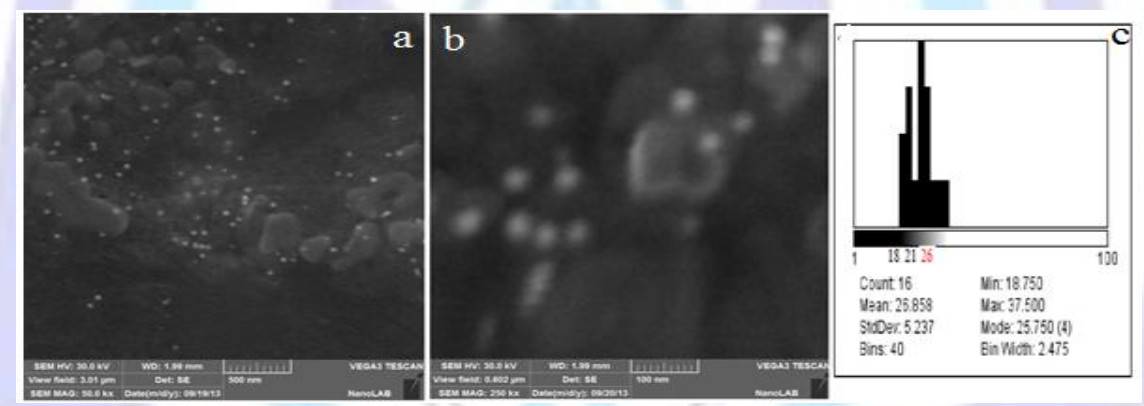

Figure4.a-SEM image b- cross section image and c-particle size distribution of the SCFX synthesized GNPs with an average size diameter $27 \mathrm{~nm}$

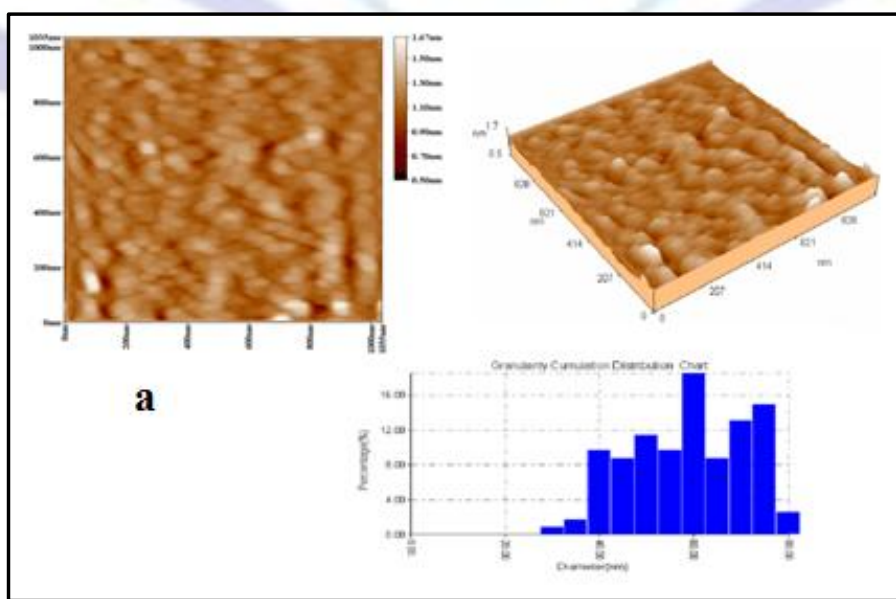

Figure 5a.AFM images andparticle size distribution charts of the cefotaxime--synthesized GNPs with an average diameter around $\mathbf{5 6 . 4 7}$ 


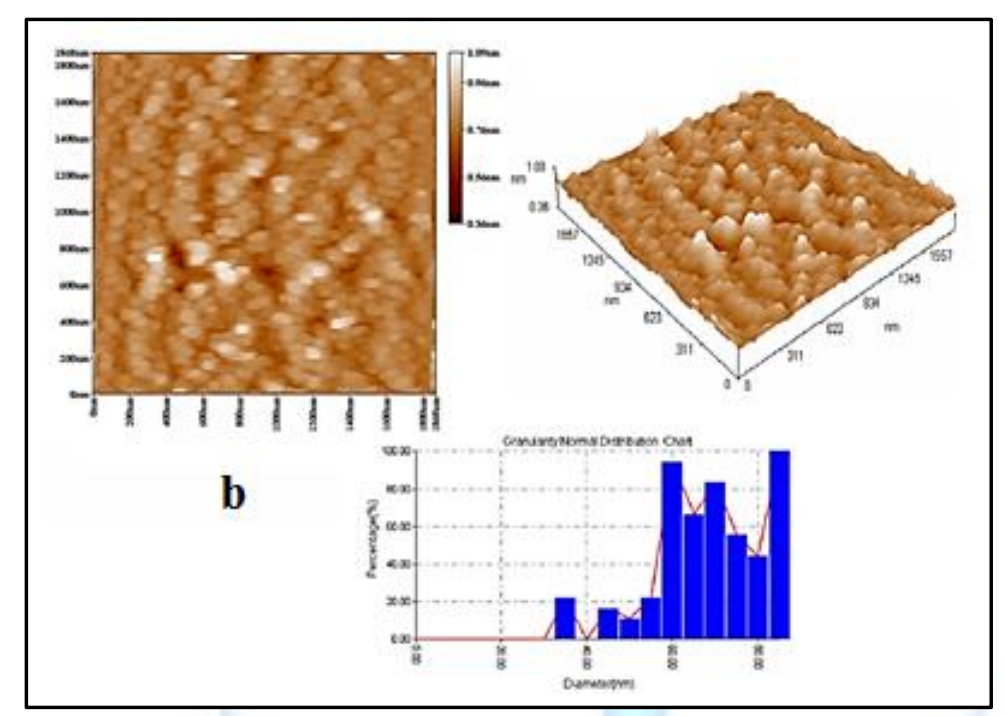

Figure 5b.AFM images andparticle size distribution charts of the SCFX-synthesized GNPs with an average diameter around $66.17 \mathrm{~nm}$

The XRD patterns of GNPs synthesized by cefotaxime and SCFXareshown in (Figures 6a and b)), respectively. Two diffraction peaks were observedat $2 \theta=(38.0547,44.531)$, and $(38.077,44.42)$ degrees for the two samples respectively corresponding to the planes (111) and (200) of facecentered cubic (fcc) Au metal crystal lattice [12,19,26].
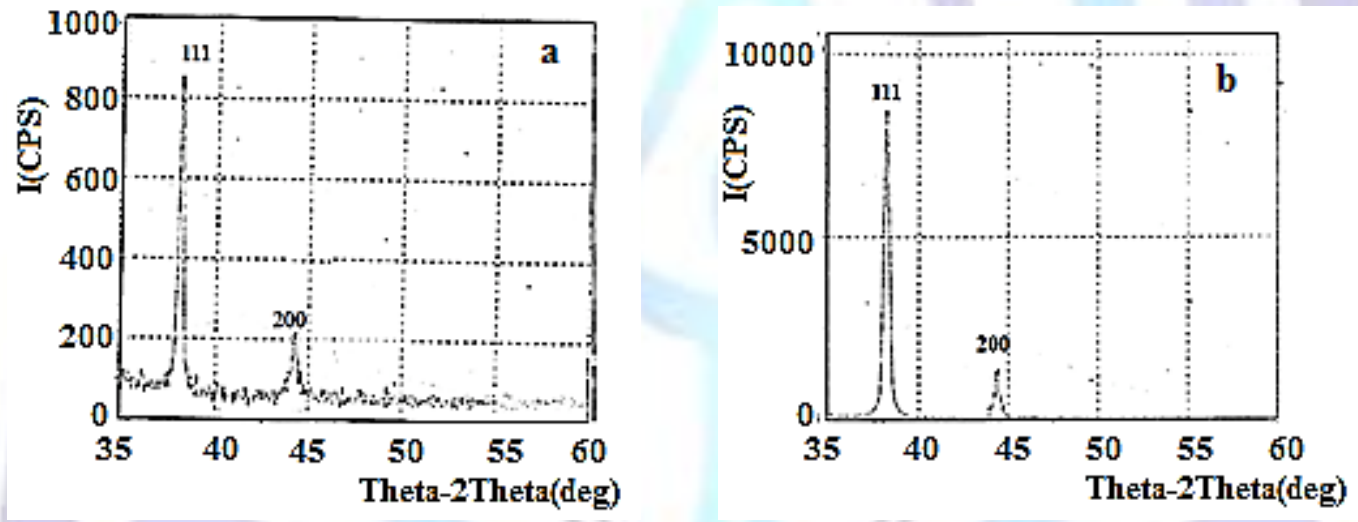

Figure 6. XRD spectrum of a-cefotaxime- , and b-SCFX- synthesized GNPs.

The FTIR spectra of CFX and CFX-capped GNPs are shown in (Figures (7a and b). respectively. The spectrum of GNPs exhibited the shifts of the two bands attributed to the $v_{a s}(\mathrm{~N}-\mathrm{H})$ and $v_{s}(\mathrm{~N}-\mathrm{H})$ vibrations of $\mathrm{NH}_{2}$ which were located at $u$ 3348 and $3252 \mathrm{~cm}^{-1}$ respectively of freeCFX[11, 27-29] were shifted to lower wavenumbers at 3250 and $3285 \mathrm{~cm}$ 1 respectively

The FTIR spectra of CFX showed that the bands assigned to the stretching vibrations of carbonyl groups of $\beta$-lactam ring , the overlapped amide and ester carbonyls carboxylate $(C=O)$ groups located at 1750,1647 [30] and 1728cm ${ }^{1}[31,32]$ respectively were shifted to 1743 , and $1721 \mathrm{~cm}^{-1}$ respectively. The bands at $1612,1388 \mathrm{~cm}^{-1}$ corresponding to the carboxylate asymmetrical and symmetrical stretching modes $[28,30,33]$ were shifted to 1635 and $1364 \mathrm{~cm}^{-1}$ respectively while the band assigned to $\mathrm{U}(\mathrm{C}-\mathrm{S})$ vibrations of the six membered thiazene ring of free $\mathrm{CFX}$ at $(593) \mathrm{cm}^{-1}[$ 35]was shifted to $570 \mathrm{~cm}^{-1}$. The FTIR spectrum of SCFX- synthesized GNPs shown in Figures (7 c and d)) respectively displayed shifts of bands attributed to the stretching vibration of both $\beta$-lactam ring carbonyl groups and amide carbonyl groups as well as C-S group observed at 1744, 1656 and $580 \mathrm{~cm}^{-1}$ respectively in the spectrum of the free ligand [27, $29,30,35,36$ ] to higher wavenumbers at 1760,1732 and $592 \mathrm{~cm}^{-1}$ respectively. The bands assigned to the asymmetric and symmetric stretching vibration, respectively of carboxylate anion, located at $(1621)$ and $(1377) \mathrm{cm}^{-1}[29,33] \mathrm{were}$ observed at $\mathrm{u} 1621$ and $1370 \mathrm{~cm}^{-1}$ respectively, while the band attributed to the azomethine group remained in its position at $1643 \mathrm{~cm}^{-1}[29,38]$. These observations confirm the conjugation of GNPs by CFX and SCFX molecules 

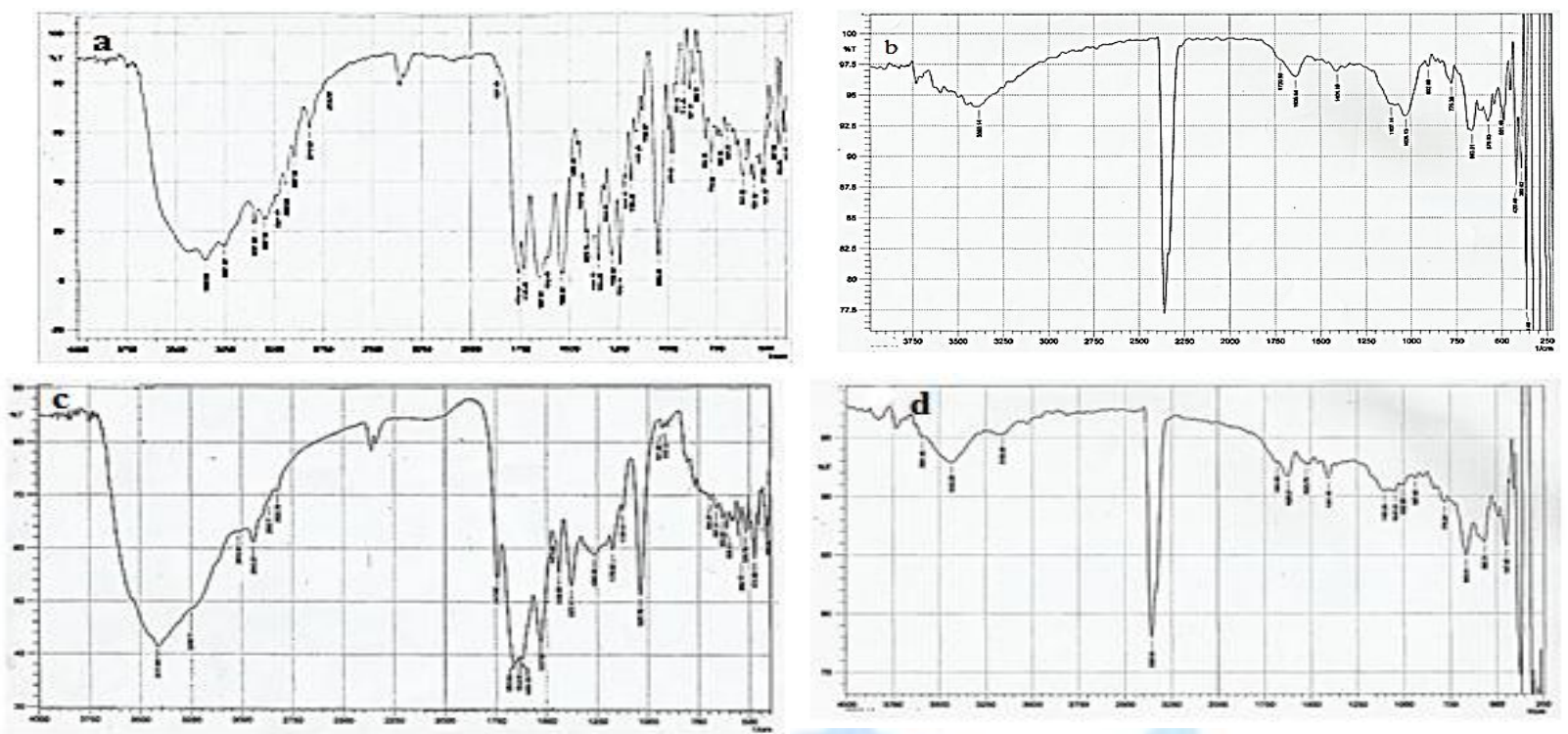

Figure7.FTIR spectra of (a)-CFX (b)-CFX-capped GNPs (c)-SCFXand (d) - SCFX-- capped GNPs.

\subsection{Concentration effect}

Figures $8(\mathrm{a}$ and $\mathrm{b})$ show the variation of intensity and position of surface plasmon band SPB of gold nanoparticles solutions prepared from adding a fixed volume $(1 \mathrm{ml})$ of cefotaxime standard solution $\left(2 \times 10^{-4} \mathrm{M}\right)$ to different volumes of $\mathrm{AuCl}_{4}{ }^{-}$standard solutions and diluted to $5 \mathrm{ml}$. The concentration ratio of $\mathrm{CTX} / \mathrm{Au}(\mathrm{III})$ ions in the resulting solution mixtures are: $3.2,1.6,1.067,0.8,0.64,0.533,0.4,0.32,0.267,0.229$ respectively (samples $1-10$ respectively). At highest concentration ratio of CTX/ $\mathrm{Au}$ (III) (3.2, sample 1) no synthesis of GNPs was observed even after $24 \mathrm{~h}$ as the solution remained colorlessand the spectrum exhibited only the weak absorption bandsof the original antibiotic.
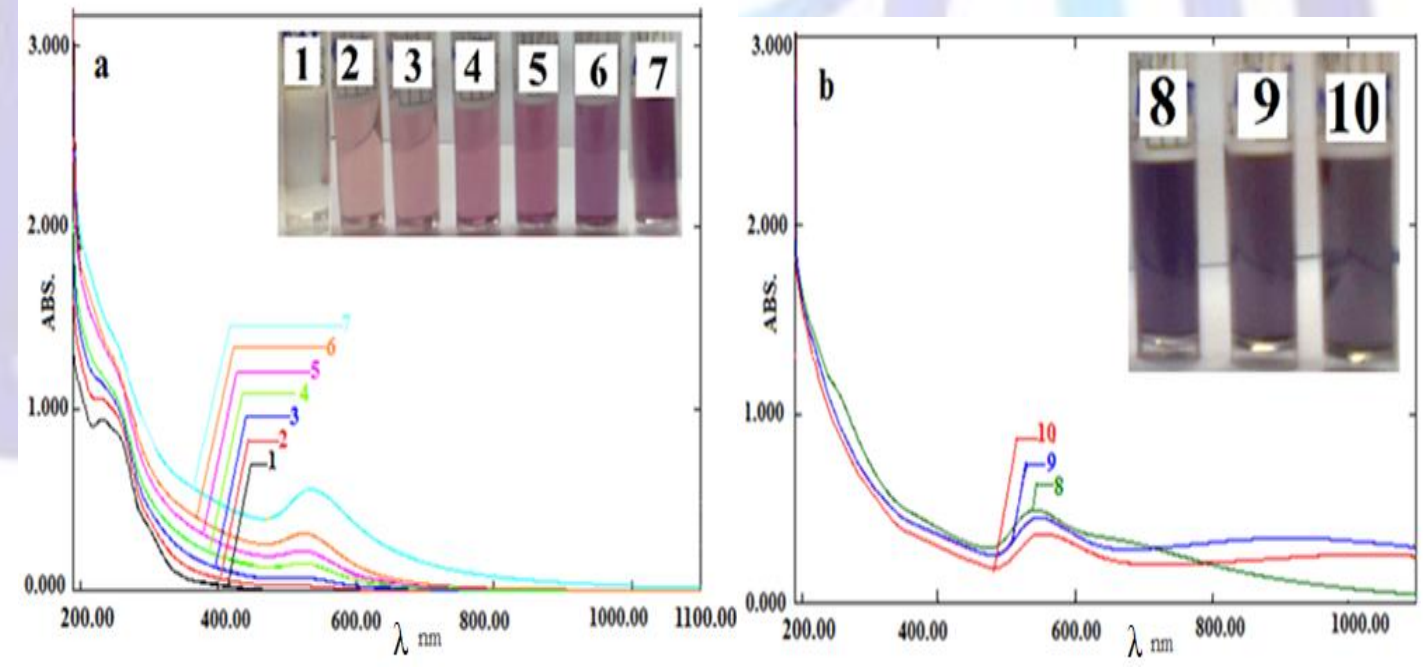

Figure 8.Absorption spectra of CFX -capped GNP at different concentrations of gold ion after $24 \mathrm{~h}$

At concentration ratios 1.6-0.4 ( samples 2-7) the colors of solutions turned ruby pink to purple within 2-3 $\mathrm{h}$ and their spectra showed increased intensity of a single absorption band observed at 520-535 nm assigned to surface plasmon resonance of spherical GNp with an estimated particle size of 38-60 nm diameter [13-15] At lower concentration ratio of $\mathrm{CTX} / \mathrm{Au}$ (III) $(0.32,0.267$ and 0.229 , samples 8-10 respectively) the colors of solutions were dark violet and their spectra displayed two absorption bands, a broad low intensity band which appeared at longer wavelength range $\lambda$ $544-556 \mathrm{~nm}$ and another band at $\lambda 680,921 \mathrm{~nm}$ respectively which refers to the formation of non-sphericalGNPs aggregates or gold nanorods[17,19,26,38]. The colloid of CTX/ Au(III) 0.64( solution 5, Figure9a) was stable for up to 60 days without significant changes in the position of SPB. The second colloid of CTX/ Au(III) 0.32 (sample 8, Figure 
(9b))showed a gradual shifts in the positions of both bands with time,then the colloid remained stable for one month. These resultsrefer to the protection and stabilization of GNPs by CFX.
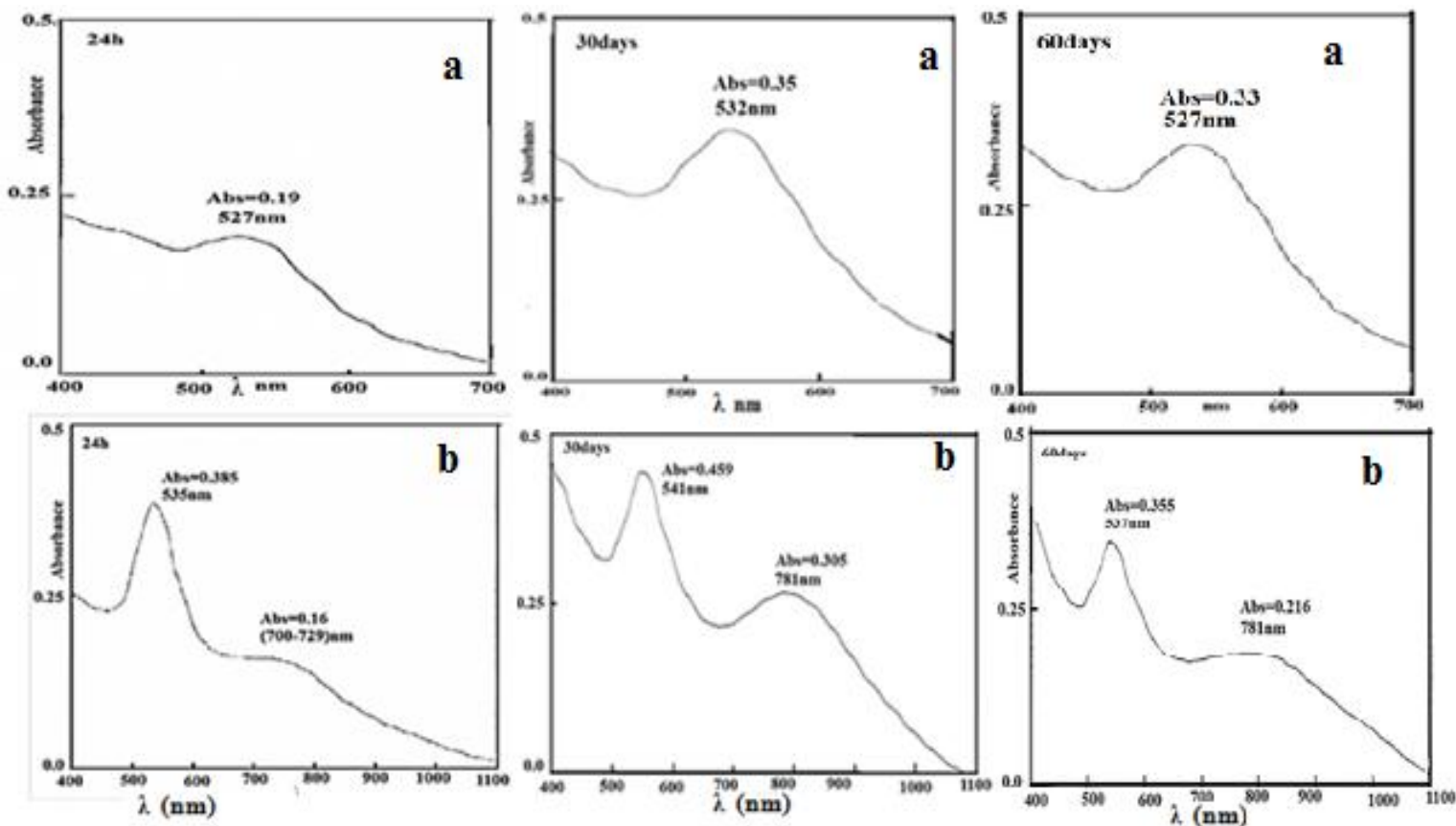

Figure 9.Absorption spectra of CFX-capped GNPs prepared from solutions containing a concentration ratio of $\mathrm{CFX} / \mathrm{Au}(\mathrm{III})=0.64$ (sample 5)(a) and 0.32 (sample 8)(b) after $24 \mathrm{~h}$ and four weeksand 60 days

Figures (10 a and b) show the variation of intensity and position of SPB of gold nanoparticles solutions prepared from adding a fixed volume of SCFX standard solution $\left(1.6 \times 10^{-4} \mathrm{M}\right)$ to different volumes of $\mathrm{AuCl}_{4}{ }^{-}$standard solutions. The concentration ratios of SCFX /Au (III)in these samples were:2.56, 1.28, 0.85, 0.64, 0.512, 0.43, 0.32, $0.256,0.213$ and 0.183 (samples 1-10). The highest concentration ratios 2.56 and 1.28 (samples 1 and 2 respectively) showed no reduction of $\mathrm{Au}$ (III) ions as the resulting solutions remained colorless and no SPBs were observed in their spectrafor $48 \mathrm{~h}$. At lower concentration ratios $(0.85,0.64,0.512,0.43$, samples 3-6 respectively), the colors of solutions became ruby pink to purple within $2 \mathrm{~h}$ and their spectra showed a single SPB appeared at wavelength range 517 $533 \mathrm{~nm}$ related to spherical GNp with estimated particle size range 32-56 nm diameter[13-15].
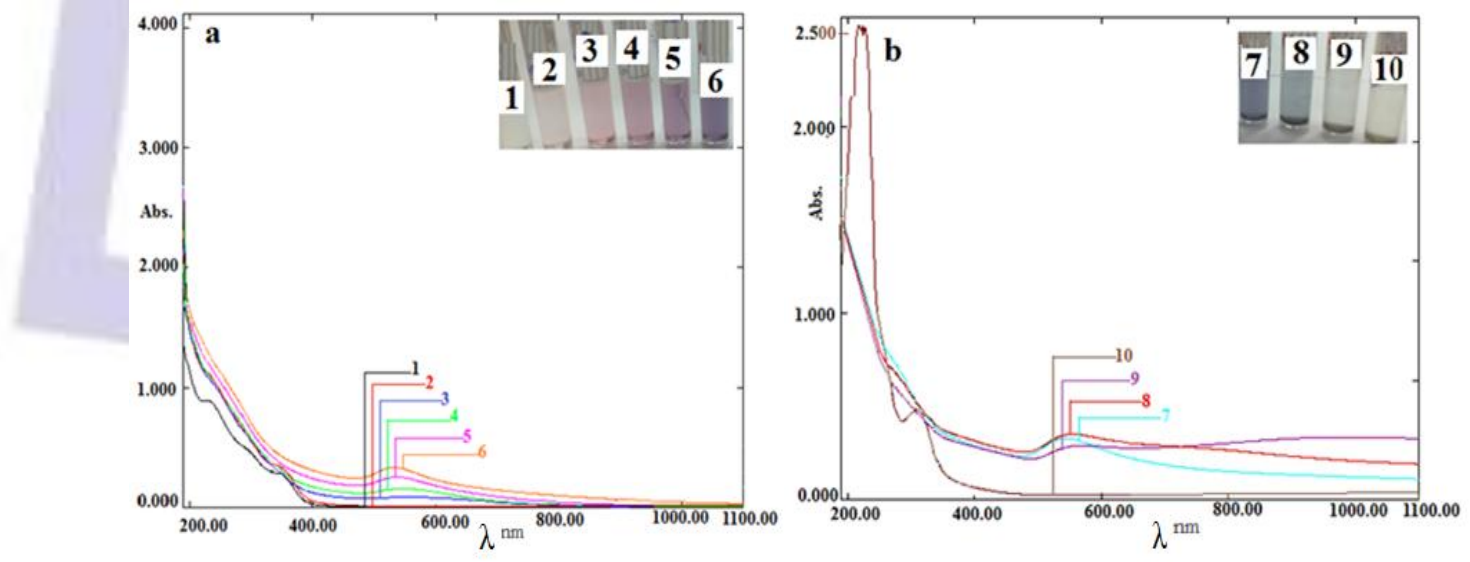

Figure 10. Absorption spectra of GNP at different concentrations ratio of SCFX /Au(III) after 24 h : a-samples(1-6), b-samples (7-10).

The spectrum of sample $4(\mathrm{SCFX} / \mathrm{Au}(\mathrm{III})=0.64)$ (Figure $(11 \mathrm{a})$ shows that maximum absorption of SPB was achieved after 4 weeks then the solution remained stable in the wavelength range 530-532 nm for 2 months. This indicates that GNPs have been capped and stabilized by SCFX. At concentration ratio 0.32 (sample 7) the SPB became broader and its position was shifted to longer wavelength $(544 \mathrm{~nm}$ ) which refers to formation of larger particle sizes (85-86) nm diameter [13-15] as a result of aggregation. At concentration ratios $0.256,0.213$ ( samples 8 and 9 respectively) the solutions were of dark violet colors and their spectra displayed a broad low intensity band at wave length range 554 and $569 \mathrm{~nm}$ respectively and another band at 740 and $1000 \mathrm{~nm}$ respectively which refers to the formation of non spherical GNPs aggregates or gold nanorods $[17,18,26,38]$. The spectrum of sample 9 (Figure $11 \mathrm{~b}[\mathrm{SCFX}] /[\mathrm{Au}(\mathrm{III})]=0.213$ ) exhibited simultaneous hypsochromic shift of the transverse plasmon band and increase in the longitudinal/transverse band ratio with time which reached its maximum value after 25 days. After two months, the solution showed hypsochromic shifts decreased intensity of both bands. At the lowest ratio of [SCFX]/[Au(III)] $(0.183$, sample 10) the 
colors of solutions were pale yellow and the resulting spectrum showed the absorption bands of $\mathrm{AuCl}_{4}{ }^{-}$which refers to incomplete or no reduction of $\mathrm{Au}$ (III) by SCFX at this ratio These results show that the concentration ratio of CTX / Au(III) and $([\mathrm{SCFX}] /[\mathrm{Au}(\mathrm{III})]$ played an important role in controlling both size and morphology of the synthesized GNps.
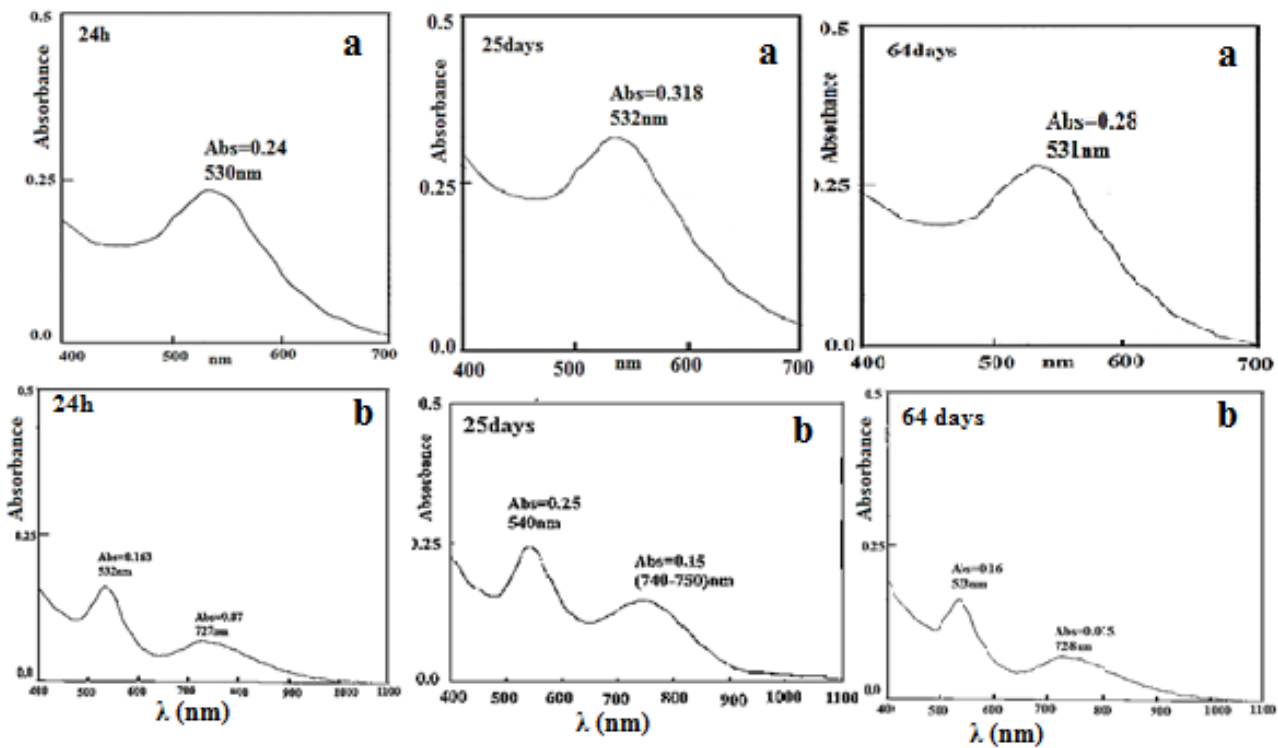

Figure 11. Absorption spectra of SCFX - conjugated GNPs containing a concentration ratio of SCFX/Au (III) = 0.64 (sample 4) and 0.213 ( sample 9) after24h, 25 days and 64 days.

\section{3. pH Effect:}

Synthesis of GNPs by CFX andSCFX was conducted using the optimum concentration ratio of CFX/Au (III) (0.64)at different $\mathrm{pH}$ media. The progress of reaction was monitored by UV-visible spectrophotometry as is shown in (Figures 12 and 13) respectively. The development of pink color as well absorption maxima of surface plasmon band (SPB) using CFX were only observed at $\mathrm{pH}=3.84$ after $3 \mathrm{~h}$. The solution exhibited one SPB at $525 \mathrm{~nm}$ characteristic of spherical GNPs [12,16-18,39]with estimated size diameter 30-40 nm [37].
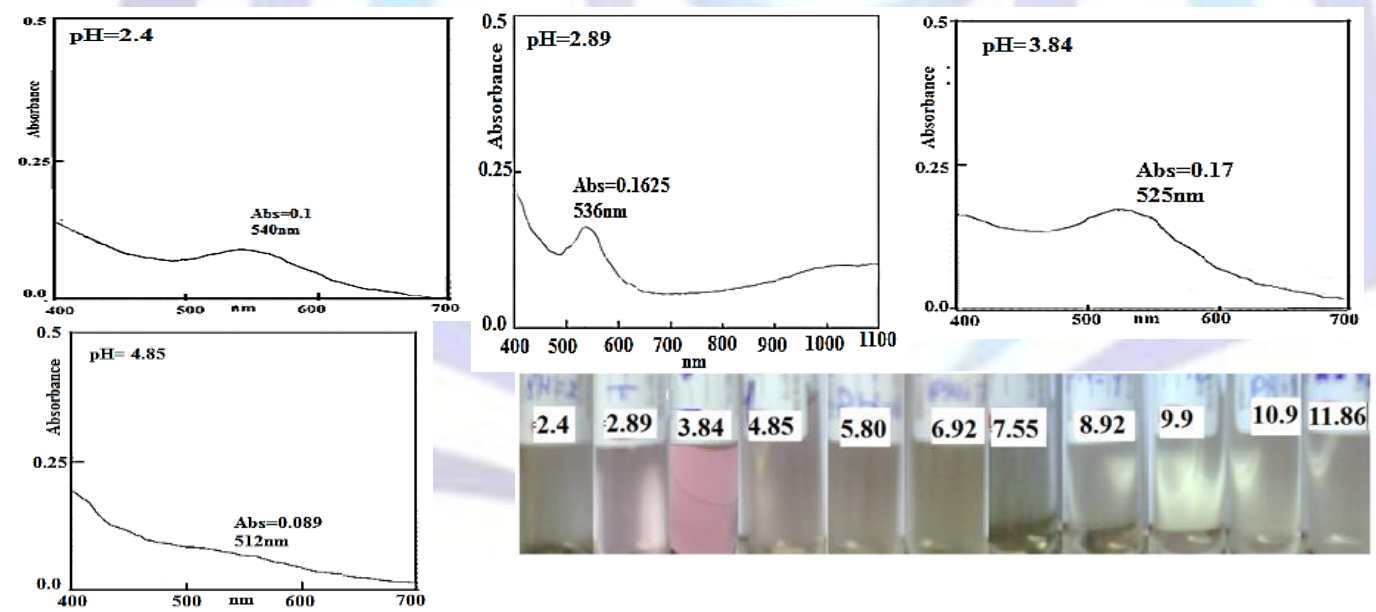

Figure 12. Absorption spectra and images of CFX synthesized GNPs solutions in different pH media at room temperature after $24 \mathrm{~h}$.

After $24 \mathrm{~h}$ the position of SPB was shifted to $532-536 \mathrm{~nm}$. The colloid was stable for almost two weeks. Changes in colors and spectra were also observed at $\mathrm{pH} 2.4,2.89$ and 4.85 (Figure (12)) while still no color changes occurred at higher $\mathrm{pH}$ values. At $\mathrm{pH}=2.89$ the solution showed a violet color and the spectrum of this colloid showed two absorption bands appeared at $536 \mathrm{~nm}$ and $1057 \mathrm{~nm}$ referring to the formation of non-sphericalGNp(nanorods) [17,26,38](Figures (12)).Figure 13 shows the UV-visible spectra of GNPs synthesized by SCFXat different pH range (2.4- 11.86). After 3h solution of $\mathrm{pH}=2.89,3.84,4.85$ and 5.80 gave changes in colors after $3 \mathrm{~h}$ and their absorption spectra exhibited the bands assigned to SPR of spherical GNPs at $\lambda 560,533,555$ and 555 respectively $\mathrm{nm}$. This indicates that the reduction of $\mathrm{Au}$ (III) ions by SCFX can be achieved at wider $\mathrm{pH}$ range. The solutions were stable for more than 1 week which refers to protection of GNPs by the ligand molecules. At $\mathrm{pH}=4.85$ the color of solution was violet and its spectrum exhibited one SPB at $555 \mathrm{~nm}$ characteristic of spherical GNPs[12,16-18,39,40]with estimated GNP diameter 60-80 nm [39] . After 24h, the spectrum exhibited gradual shifts of the band position to shorter wavelength $(530-535 \mathrm{~nm})$ with a gradual appearance of additional absorption band appeared $\lambda 720-730 \mathrm{~nm}$ referring to the formation of elongated GNPs. The above mentioned 
results show that the variation of $\mathrm{pH}$ plays has an important effect on the rate of $\mathrm{Au}$ (III) ions reduction by the CFX and SCFX to GNPs as well as on the size and morphology of GNPs which may be attributed to the difference in acid-base behavior of the functional groups present in the skeleton of the two molecules.

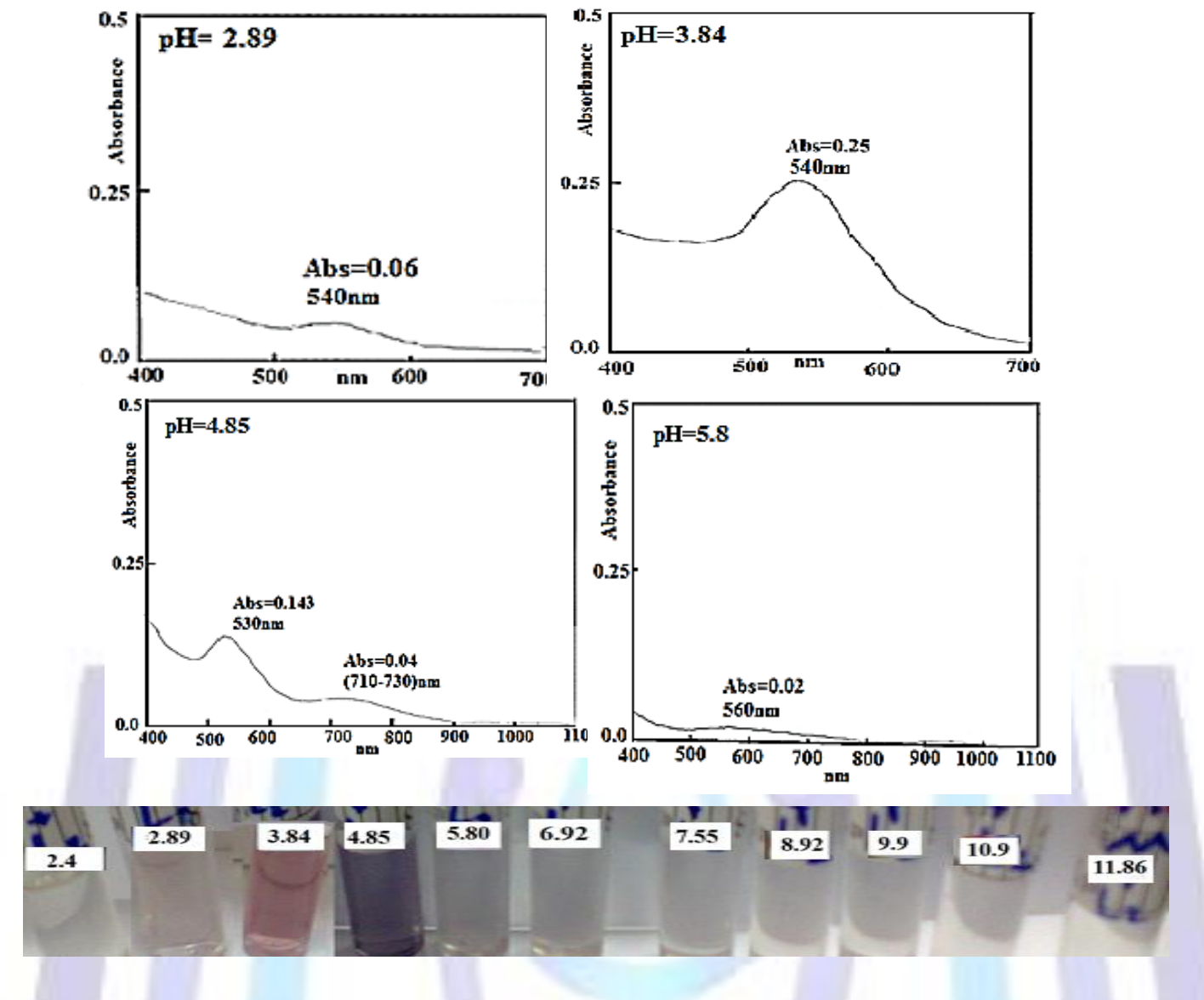

Figure 13. Variation of surface plasmonresonance bands of SCFX- synthesized GNPs prepared in different pH media at room temperature after $24 \mathrm{~h}$.

\subsection{Temperature effect}

Figures 14and15 show respectively the progress GNPs synthesis and color change images with time for solution mixtures containing equal volumes of CFX and SCFXwithAu(III) standard solutions at temperature range $20-80{ }^{\circ} \mathrm{C}$ monitored by uv-visible spectroscopy. At $20^{\circ} \mathrm{C}$ the reduction process of $\mathrm{Au}(\mathrm{III})$ by both ligands was considerably slow and the pink color was detected after several hours. Gradual increaseofheating temperature up to $80^{\circ} \mathrm{C}$ caused increased rates of GNPs formation accompanied with increased amplitude of SPBs. This indicates that increased reaction temperature enhancedthe reduction process,and the growth of nanoparticles as a result of high rate of nucleation [41].

The reduction progress with temperature led to bathochromic shifts of SPBs without changing the profile of these bands. The optimum heating temperature for production of more stabilized GNPs solutions by CFX and SCFX with time was $40{ }^{\circ} \mathrm{C}$ for 15 and 10 min respectively. Despite the high rate of GNPS synthesis at (70 and $\left.80{ }^{\circ} \mathrm{C}\right)$ the resulting colloids showed gradual formation of sediments with time. This may be attributed to the desorption or catalytic decomposition of the capping ligand which leads to further aggregations of GNPs. The above mentioned results showed the importance of temperature as an additional controlling factor on the particle sizes and stability of the studied GNPs solutions. 

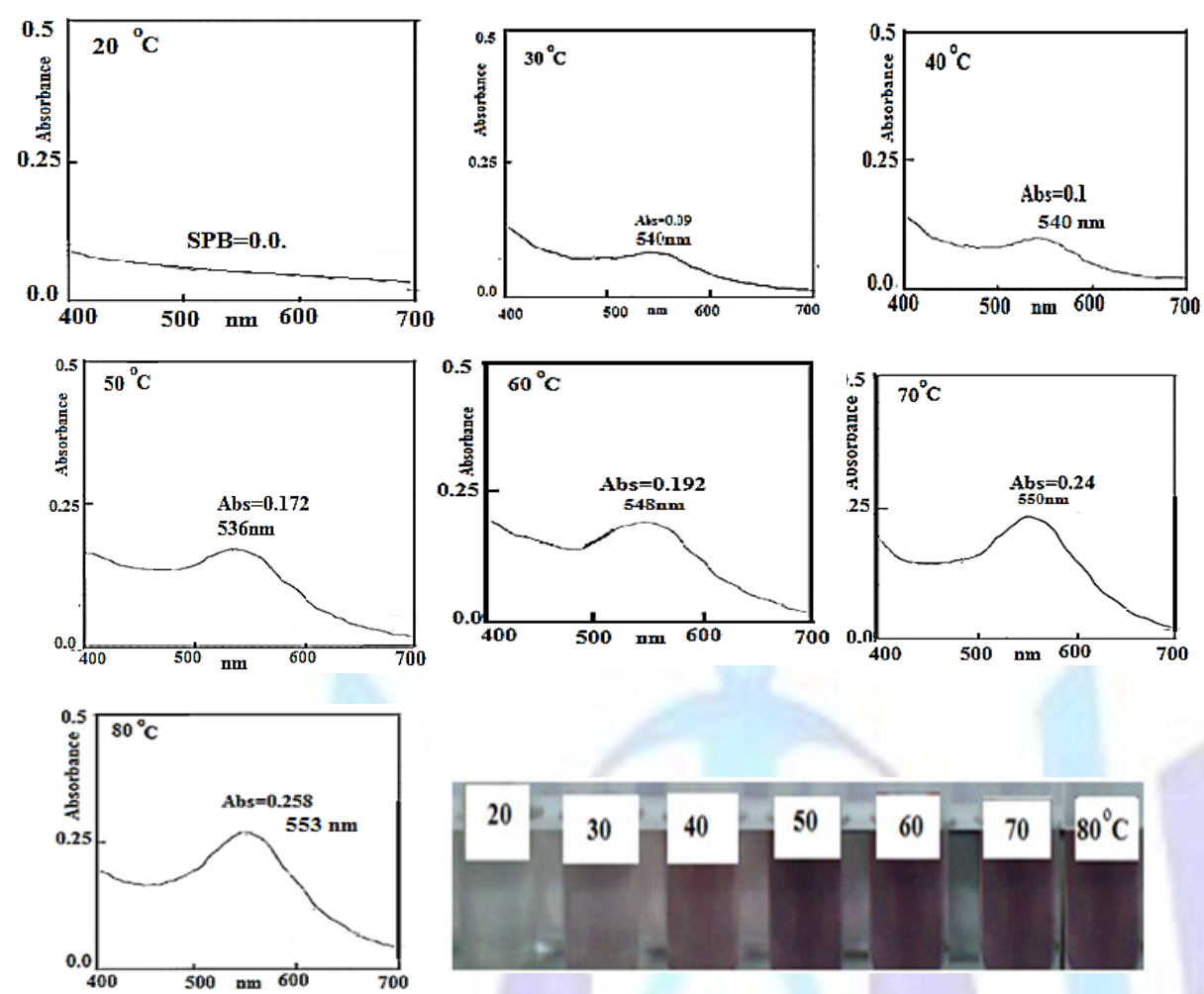

Figure14. Spectral changes and images of CFX- synthesized spherical GNPs at $20-80^{\circ} \mathrm{C}$.
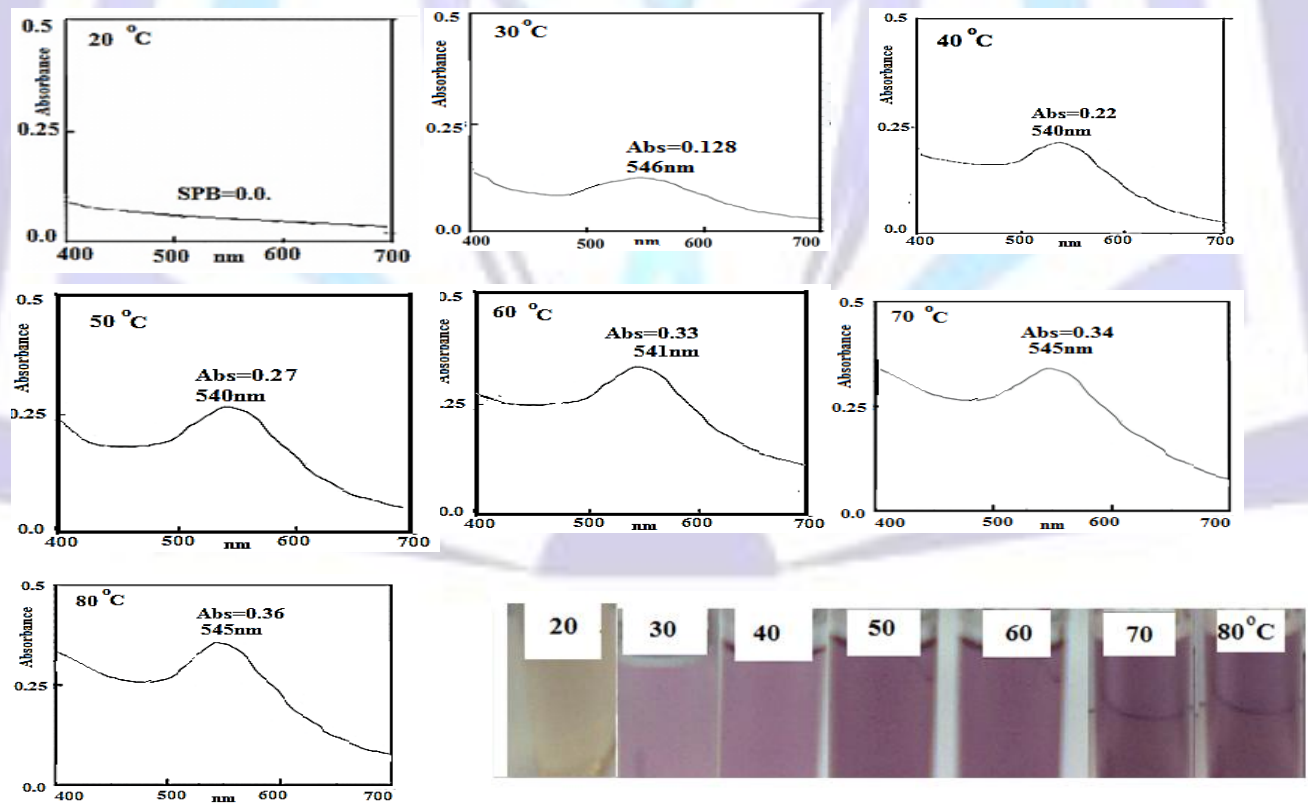

Figure 15.Spectral changes andimages of SCFX.synthesized GNPs solutions prepared at $20-80^{\circ} \mathrm{C}$

\section{CONCLUSIONS}

Gold nanoparticles have been successfully produced by a simple wet chemical reduction of $\mathrm{AuCl}_{4}{ }^{-}$in aqueous solutions using cefotaxime antibiotic, and the cefotaxime Schiff base SCFX without the use of other reducing or dispersing agents. Morphology and sizes of the synthesized gold nanoparticles for the studied ligands were detected from the positions and extinction of surface plasmon bands monitored by UV-visible spectrophotometry and confirmed by SEM, TEM and AFM analysis. Conjugation of GNPs by the two ligands was confirmed by FTIR spectroscopy. The shape, size 
of GNPs and enhancement of GNPs synthesis are controlled by reactant and its concentrations, $\mathrm{pH}$, temperature and heating time intervals. The higher synthesis rates of GNPs by the Schiff base ligand suggested that oxidation of amino group was not totally responsible for the reduction of $A u(I I I)$ ion, but may be further attributed to the oxidation of the lactam ring, ,thiazine ring groups, carboxylate anion as well as azomethine group of the Schiff base ligand .

\section{REFERENCES}

[1]JagannathanA., Surface Functionalisation of Nanoparticles and Their Biological Applications Chapter 3 - Surface functionalisation of nanoparticles using biomolecules" University of Pune, (2011) , 46-94.

[2]DemurtasM.,Perry C. C., Facile one-pot synthesis of amoxicillin-coated goldnanoparticles and their antimicrobial activity. Gold Bull, 47, (2014),103-107.

[3]Bhattacharya D., Saha B., Mukherjee A., Santra C. R.,Karmakar P.,Gold Nanoparticles Conjugated Antibiotics: Stability and Functional Evaluation. Nanoscience and Nanotechnology, 2(2), (2012), 14-21.

[4]Shenoy W., Fu J., L, C., CrastoG., .Jones C.,DiMarzioS., Sridhar M.Amiji M , Surface functionalization of gold nanoparticles using hetero-bifunctional poly(ethylene glycol) spacer for intracellular tracking and delivery. International Journal of Nanomedicine:1(1), (2006), 51-57.

[5]PongsuchartM., Danladkaew C., Khomvarn T.,Sereemaspun A, Effect of Glutathione-Stabilized Gold Nanoparticles in 3T3 Fibroblast Cell. International Conference on Clean and Green Energy IPCBEE,27,(2012) IACSIT Press, Singapore,

[6]Savi G. D., Trombin A. C.,Generoso J. S. ,BarichelloT.,PossatoJ. C.,Ronconi,J. V. V., da Silva Paula, M. M., Antibacterialactivity of gold and silver nanoparticles Impregnated withantimicrobial agents.RevistaSaúde e Pesquisa, 6(2), (2013), 227-235.

[7] Ra A., PrabhuneA., Perry, C. C.,Antibiotic mediated synthesis of gold nanoparticles with potent antimicrobial activity and their application in antimicrobial coatings"J. Mater. Chem., 20, (2010), 6789-6798.

[8]Hussain R. K.,Synthesis and characterization of some new Schiff base derivatives of cefotaxime with their metal complexes and study the reduction of $\mathrm{Au}$ (III) ions to goldnanoparticles. MSc Thesis, University of Baghdad,College of Science, Baghdad ,Iraq.(2014).

[9]Link S.El-Sayed M. A.,Size and temperature dependence of the plasmon absorption of colloidal gold nanoparticles. J. Phys. Chem. B, 103, (1999), 4212-4217.

[10] Jiang G., WangL..Chen W., Studies on the preparation and characterization of gold nanoparticles protected by dendrons. Materials Letters,61, (2007), 278-283.

[11] Silverstein, R. M., Webster, E. X. and. Kiley, D. J.. Spectrometric Identification of Organic Compounds 7th Edn ,John Wiley and Sons. Inc., Hoboken, USA,(2005), 1-502.

[12]Das, R.,Nath, S. S. and Bhattacharjee R... Optical properties of linoleic acid protected gold nanoparticles.JournalofNanomaterials, 2011,ID 630834. (2011),1-4

[13]Slouf M., KuzelR.. MatejZ..Preparationandcharacterization of isomeric gold nanoparticles with pre-calculated size.Z. Kristallogr.,23, (2006). 319- 324.

[14]Martínez J. C., Chequer N. A., GonzálezJ. L., Cordova T., Alternative methodology for gold nanoparticles diameter characterization using PCA technique and uv-vis.spectrophotometry.Nanoscience and Nanotechnology,2(6), (2012), 184-189.

[15]Jain L.,El-SayedM., Calculated absorption and scattering properties of gold nanoparticles of different size, shape, and composition: applications in biological imaging and biomedicine. J. Phys. Chem. B, 110, (2006), 7238-7248.

[16]Bhumakar D. R., Joshi H. M.,Sastry M.,PokharkarV. B., Chitosan reduced gold nanoparticles as novel carriers for transmucosal delivery of insulin. Pharmaceutical Research,24(8),(2007), 1415-1426 .

[17]Boopathi S., SenthilkumarS., Phani K. L., Facile and one Pot synthesis of gold nanoparticles using tetraphenylborate and polyvinyl pyrrolidone for selective colorimetricdetection of mercury ions in aqueous medium. Journal of Analytical Methods in Chemistry, 2012, ID 348965 ,(2012), 1-6.

[18]Ghosh D., Sarkar D.Girigoswami A., Chattopadhyay N., A fully standardized method of synthesis of gold nanoparticles of desired dimensions in the range 15-60 nm. Journal of Nanoscience and Nanotechnology,11(2), (2011), 1141-1146.

[19]Johan M. R., Chong L. C. HamiziN. A., Preparation and stabilization of monodisperse colloidal gold by reduction with monosodium glutamate and poly (methylmethacrylate).,Int. J. Electrochm. Sci., 7, (2012), 4567-4573.

[20]Xavier B., RamanandA., SagayarajP., Investigation on a facile one-pot rapid synthesis approach for developing modestly monodispersed and stable spherical gold nanoparticles, Der PharmaChemica, 4(4), (2012), 1467-1470.

[21]Tom R. T., Suryanarayanan V., Reddy P. G.,Baskaran S.,PradeepT., Ciprofloxacin-protected gold nanoparticles.Langmuir, 20, (2004), 1909-1914. 
[22]SubramaniamC., Tom R. T., Pradeep T., On the formation of protected gold nanoparticles from $\mathrm{AuCl}_{4}{ }^{-}$by the reduction using aromatic amines. Journal of Nanoparticle Research, 7( 2-3), (2005), 209-217.

[23]Hari N., Thomas T. K., Nair A. J , Comparative study on the synergistic action of garlic Synthesized and citrate capped silver nanoparticles with $\beta$-Penemantibiotics.Nanotechnology, 2013, ID $792105,(2013), 1-6$.

[24]Li, P., Li, J. , Wu, C., Wu, Q. and Li, J.(2005). Synergistic antibacterial effects of $\beta$-lactam antibiotic combined with silver nanoparticles, Nanotechnology, 16, pp:1912-1917.

[25]Jagannathan R., PoddarP., PrabhuneA., Cefalexine mediated synthesis of quasi-spherical and anisotropic gold nanoparticles and their in situ capping by the antibiotic. J. Phys. Chem. C, 111(2009), 6933-6938.

[26]Wang W., Chen Q., Jiang C. , YangD., Liu X.,Xu S., One-step synthesis of biocompatible gold nanoparticles using gallic acid in the presence of poly-(N-vinyl-2-pyrrolidone). Colloids and Surfaces A: Physicochem. Eng. Aspects ,301,(2007), 73-79.

[27] AnaconaJ. R., Lopez M., Mixed-ligand nickel (II) complexes containing sulfathiazole and cephalosporin antibiotics: synthesis, characterization, and antibacterial activity. Int. Journal of Inorganic Chemistry, 2012, ID 106187 (2012),18.

[28]AnaconaJ. R., Osoriol., Synthesis and antibacterial activity of copper(II) complexeswithsulphathiazole and cephalosporin ligands. Transition Met Chem,33(4), (2008), 517-521.

[29]Anacona J. R., Calvo J., Almanza O. A., Synthesis, spectroscopic and magnetic studies of mono- and polynuclear Schiff base metal complexes containing salicilydene-cefotaxime ligand $\left(\mathrm{H}_{2} \mathrm{~L}\right)$. International Journal of Inorganic Chemistry, 2013,ID 108740,(2013), 1-7.

[30]AnaconaJ.R., Silva, G. D., Synthesis and antibacterial activity of cefotaxime metal complexes.J. Chil.Chem.Soc.,50(2),(2005), 1-9.

[31]Sultana N., ArayneM. S., AfzalM., Synthesis and antibacterial activity of cephradine metal complexes: part II complexes with cobalt ,copper ,zinc and cadmium. Pakistan Journal of Pharmaceutical Sciences, 18(1),(2005), 3642.

[32]Kumar V. J., GuptaP. B. , Pavan Kumar K.S.R, PrasadaRao, K.V.V,. Prasanna S. J, Siva Kumar G.S. , Sharma H. K., Mukkanti K., Identification and characterization of new impurity in Cefotaxime Sodium drug substance. Der PharmaChemica, 2(3), (2010), 230-241.

[33]MannaS. C.,Mistr S. A.,DipankaJ. A., Supramolecular assembly involving ion pairs of coordination complexes with a host-guest relationship: synthesis, crystal structure, photoluminescence and thermal study.Cryst. Eng. Comm. Royal Soc. Chem. 14(21), (2012), 7415-7422.

[34]Meyers R. A, Interpretation of Infrared Spectra, A Practical Approach. John Wiley and Sons Ltd, Chichester, (2000), .

[35]El-SaidA. I. ,AlyA. A. M., El-MeligyM. S., IbrahimM. A., Mixed ligand zinc (II) and cadmium(II) complexes containing ceftriaxone or cephradine antibiotics and different donors. J. Argent. Chem. Soc., 97(2), (2009), 149-165.

[36]SultanaN. ,AraynaM. S., AfzalM., Synthesis and antibacterial activity of cepharadine metal complexes :Part I: complexes with magnesium, calcium, chromium and manganese , Pakistan Journal of Pharmaceutical Sciences, 16(1), (2003), 59-72.

[37]Bukhar, I. H. ,ArifM., Akbar J., Khan A. H., Preparation, characterization and biological evaluation of Schiff Base transition metal complexes with cephradine, Pakistan Journal of Biological Sciences, 8(4), (2005), 614-617.

[38] Samal A. K., Sreeprasad T. S, Investigation of the role of $\mathrm{NaBH}_{4}$ in the chemical synthesis of gold nanorods. J. Nanoparticle Research, 12(5), (2010), 1777-1786.

[39]ShiW., Casas J., Venkataramasubramani M., Tang L. , Synthesis and characterization of gold nanoparticles with plasmon absorbance wavelength tunable from visible to near infrared region. ISRN Nanomaterials, 2012, ID 659043 (2012), $1-9$.

[40 LongN. N., L. V. Vu, Ch. D. Kiem, S. C. Doanh, C. Th. Nguyet, Ph. Th. Hang, N. D. ThienandQuynh,L.Synthesis and optical properties of colloidalgold nanoparticles. (2009). Workshop on Advanced MaterialsScience and Nanotechnology published in J. of Phys. Conference Series ,187, 012026, (2009) , 1- 8.

[41]SardarR., Shumaker-Parry J. S., Spectroscopic and microscopic investigation of gold nanoparticle formation: ligand and temperature effect on rate and particle size. J. Amer. Chem. Soc., 133(21), (2011), 8179-8190. 